\title{
Long non-coding RNA AB209630 suppresses cell proliferation and metastasis in human hepatocellular carcinoma
}

\author{
TENG LI ${ }^{1}$, YUN LIU ${ }^{2}$ and YANMING SUN ${ }^{1}$ \\ Departments of ${ }^{1}$ Interventional Radiology and ${ }^{2}$ Hematology, \\ The People's Hospital of Weifang, Weifang, Shandong 261041, P.R. China
}

Received April 19, 2016; Accepted March 24, 2017

DOI: $10.3892 /$ etm.2017.4927

\begin{abstract}
Hepatocellular carcinoma (HCC) is one of the most prevalent cancers worldwide and the second most common cause of cancer-related mortalities. With a high potential for metastasis and recurrence, HCC is refractory to cure. The present study aimed to explore the role of a recent-discovered LncRNA, AB209630, in human HCC, in order to provide new insights useful for clinical HCC diagnosis and treatment. Reverse transcription-quantitative polymerase chain reaction was performed to examine the expression of AB209630 in clinical HCC samples and the adjacent non-cancerous tissues. The reduced expression of AB209630 observed in HCC tissues and cultured HCC cells compared with normal hepatic tissues and cells prompted the construction of an AB209630-expressing plasmid with a CBP tag on the plasmid backbone. Cell proliferation and colony formation assays were conducted to detect the effects of AB209630 on HCC cell proliferation. In addition, Transwell assay and wound-healing assays were performed, the results of which further indicated that the overexpression of AB209630 inhibited the migration and invasion of HCC cells. These results revealed the inhibitory effects of AB209630 on HCC progression, and suggest the potential of AB209630 as an inhibitor of HCC for clinical use.
\end{abstract}

\section{Introduction}

Liver cancer is a common disease, particularly in Asia and parts of sub-Saharan Africa, and may occur as the result of an infection several decades earlier (1). It is the sixth most frequently occurring cancer worldwide; in 2012, there was an estimated morbidity of 782,000 cases, and the number of mortalities due to liver cancer was estimated to be $\sim 746,000$ (2). Notably, liver cancer is the second most common cause of cancer-related

Correspondence to: Dr Yanming Sun, Department of Interventional Radiology, The People's Hospital of Weifang, 151 Guangwen Street, Weifang, Shandong 261041, P.R. China E-mail: yanmingsun8912@sina.com

Key words: long non-coding RNA, AB209630, proliferation, metastasis, liver cancer mortalities worldwide $(3,4)$. Liver cancer can be divided into three categories, namely hepatocellular carcinoma (HCC), intrahepatic cholangiocarcinoma and mixed hepatocarcinoma, according to the origin of the cancer, among which HCC is the most common (5). Despite the great progress made in understanding the tumorigenesis of the disease and therapeutic options in previous decades, $\mathrm{HCC}$ continues to respond poorly to traditional therapies and relapses shortly following clinical treatment $(6,7)$. Moreover, its potential for metastasis and intrahepatic transfer makes it challenging to cure (8). Therefore, the identification of novel approaches to diagnose and treat this disease is urgently necessary.

Long non-coding RNA (LncRNA) is a class of non-protein coding transcripts of $>200$ nucleotides and $<100 \mathrm{~kb}(9)$. LncRNAs are reported to regulate the expression of genes in the biological processes of differentiation, proliferation, metastasis, reprogramming of induced pluripotent stem cells and apoptosis $(10,11)$ by interacting with chromatin-modifying enzymes (12), RNA processing (13) and structural scaffolds (14). LncRNAs have also been demonstrated to play significant roles in the tumorigenesis of HCC. For example, Zhou and Gao found that BRAF-activated non-coding RNA contributed to $\mathrm{HCC}$ initiation and progression by upregulating cell proliferation and metastasis, in addition to promoting cell apoptosis (15). Esposti et al detected 5,525 LncRNAs across various tissue types and found 57 differentially expressed LncRNAs in HCC, all of which were co-expressed with genes involved in liver metabolism, cell cycle regulation and the transforming growth factor $\beta 1$ signaling pathway (16).

AB209630 is a recently-discovered LncRNA, which was demonstrated to have a notable role in hypopharyngeal squamous cell carcinoma (HSCC) in a previous study (17). In that study, the expression of LncRNA AB209630 was found to be markedly decreased in HSCC tissues in comparison with the adjacent non-cancerous tissue. Overexpression of AB209630 was observed to inhibit cell proliferation and colony formation, and also to suppress cell metastasis. Furthermore, AB209630 promoted cell apoptosis and was associated with the prognosis of patients with HSCC. However, the detailed role of AB209630 in HSCC and the potential effects of AB209630 on other types of tumor remain unknown.

The present study explored the role of AB209630 in HCC in vivo and in vitro. Firstly, the expression of AB209630 in clinical HCC tissues and cultured HCC cells was 
evaluated. Subsequently, an AB209630-expressing plasmid was constructed using a V51 pIRESpuro-GLUE empty vector (pGLUE) containing a CBP-tag ( $\mathrm{N}$ terminal on the plasmid backbone) and the effects of AB209630 overexpression were explored. The results of this study may provide new information useful in the diagnosis and treatment of patients with HCC.

\section{Materials and methods}

Human tissues. In this study, 40 patients $(45 \pm 5$ years old, male:female $=22: 18$ ) with HCC were enrolled by the Department of Interventional Radiology of the People's Hospital of Weifang (Weifang, China). Prior to the surgery, none of the patients had received chemotherapy or radiotherapy and after the surgery, the HCC tissues and their adjacent non-cancerous tissues were frozen in liquid nitrogen immediately. Written informed consent was obtained from each patient and the study was approved by the Ethics Committee of the People's Hospital of Weifang.

Cell culture. THLE-3 human normal liver cells and the HCC cell lines MHCC97-L, MHCC97-H and HCCLM3 were purchased from American Type Culture Collection (Manassas, VA, USA) and grown in Dulbecco's Modified Eagle's Medium (DMEM; Gibco; Thermo Fisher Scientific, Inc., Waltham, MA, USA) supplemented with $10 \%$ fetal bovine serum (FBS; Gibco; Thermo Fisher Scientific, Inc.). During the study, cells were cultured at $37^{\circ} \mathrm{C}$ in a humidified incubator containing $5 \% \mathrm{CO}_{2}$. The culture medium was replaced every other day, or as stated.

$R N A$ isolation and reverse transcription-quantitative polymerase chain reaction ( $R T-q P C R)$. Total RNA from clinical tissues and cultured HCC cells was extracted using TRIzol reagent (Thermo Fisher Scientific, Inc.) and quantified using a Nanodrop 2000 instrument (Thermo Fisher Scientific, Inc.) according to the optical density values at 260 and $280 \mathrm{~nm}$ in each sample. Complementary DNA (cDNA) was synthesized using PrimeScript Reverse Transcriptase (Takara Biotechnology Co., Ltd. Otsu, Japan) and $10 \mathrm{ng}$ total RNA with the following protocol: $37^{\circ} \mathrm{C}$ for $15 \mathrm{~min}$ and $85^{\circ} \mathrm{C}$ for $5 \mathrm{sec}$. qPCR was performed with a SYBRgreen kit (Takara Biotechnology Co., Ltd.) using an ABI7900 machine (Applied Biosystems; Thermo Fisher Scientific, Inc.) in a volume of $10 \mu l$ according to the manufacturer's instructions. The thermocycling conditions were as follows: $95^{\circ} \mathrm{C}$ for $5 \mathrm{~min}$, followed by 40 cycles of $95^{\circ} \mathrm{C}$ for $15 \mathrm{sec}$ and $60^{\circ} \mathrm{C}$ for $30 \mathrm{sec}$. The primers used were as follows (17): AB209630 forward: 5'-GGGCTATTGTCCCTA AGTTGAT-3' and reverse, 5'-TGTCTTGTAGAGCATAAG GAAACC-3'; GAPDH forward, 5'-GGGAAACTGTGGCGT GAT-3' and reverse, 5'-GAGTGGGTGTCGCTGTTGA-3'. All quantitative data were quantified using the $2^{-\Delta \Delta \mathrm{Cq}}$ method (18).

Plasmid construction and transfection. pGLUE empty vector was purchased from Addgene, Inc. (Cambridge, MA, USA). Restriction enzyme sites for $E c o \mathrm{RV}$ and $B a m \mathrm{H} 1$ were used to clone AB209630. The full-length DNA sequence of human AB209630 was cloned from cultured 293T cells with PCR. The AB209630 sequence was connected to the pGLUE vector with DNA ligase for $2 \mathrm{~h}$ at room temperature. Afterwards, the combined plasmid was confirmed by sequencing (Jieli Co., Shanghai, China). Prior to the experiments,
AB209630-expressing plasmid was transfected into HCCLM3 cells using Lipofectamine 2000 (Thermo Fisher Scientific, Inc.) according to the manufacturer's protocol. HCCLM3 cells transfected with empty vector and untransfected cells served as the vector and control groups.

Western blot analysis. Protein expression was evaluated by western blot analysis. Briefly, total protein from HCCLM3 cells was collected using lysis buffer (NP-40, Beyotime Institute of Biotechnology, Nantong, China) on ice according to the manufacturer's protocol and quantified using Bio-Rad protein assay reagent (Bio-Rad Laboratories, Inc., Hercules, CA, USA). Equal amounts of protein $(40 \mu \mathrm{g})$ were loaded onto $10 \%$ SDS-PAGE for electrophoresis and then transferred to a nitrocellulose membrane (EMD Millipore, Bedford, MA, USA). The membrane was blocked for $2 \mathrm{~h}$ with $5 \%$ skimmed milk at room temperature and then incubated with primary antibodies overnight at $4^{\circ} \mathrm{C}$. The primary antibodies against CBP-tag (sc-1211, 1:1,000) and GAPDH (sc-32233, 1:1,000) were purchased from Santa Cruz Biotechnology, Inc. (Dallas, TX, USA). The membrane was then incubated with secondary goat-anti-rabbit (sc-2004) and goat-anti-mouse (sc-2005) antibodies (Santa Cruz Biotechnology, Inc.) for $1 \mathrm{~h}$ at $37^{\circ} \mathrm{C}$ with a dilution of 1:1,000. Finally, the proteins were quantified using ECL Prime Western Blotting Detection reagent (GE Healthcare, Parsippany, NJ, USA) and an ImageQuant LAS 4000 Mini Biomolecular Imager (GE Healthcare).

Cell proliferation. HCCLM3 cells were seeded in a 96-well plate at a concentration of $1 \times 10^{3} /$ well. After $24 \mathrm{~h}$, cells were transfected with AB209630-expressing plasmid or control (empty) plasmid. Cell proliferation was examined every day in the consecutive 5 days using a CellTiter 96 AQueous Non-Radioactive Cell Proliferation kit (Promega Corporation, Madison, WI, USA) following the manufacturer's protocol. The cell proliferation rate was determined by measuring the absorbance at $490 \mathrm{~nm}$ using a microplate reader (Tecan, Männedorf, Switzerland).

Colony formation assay. HCCLM3 cells were transfected with AB209630-expressing plasmid or control (empty) plasmid and cultivated in six-well plates at a density of 200 cells/well. The culture medium was changed every 2 days. After 2 weeks, the cell colonies that contained $>50$ cells were counted by staining with $0.5 \%$ crystal violet and observation under a light microscope with a magnification of $\mathrm{x} 200$.

Transwell assays. For cell migration assays, HCCLM3 cells were trypsinized and collected by low-speed centrifugation $\left(840 \times \mathrm{g}, 4^{\circ} \mathrm{C}\right)$ with serum-free DMEM. A total of $1 \times 10^{4}$ cells $(\sim 150 \mu \mathrm{l})$ were transferred into the upper chamber. The lower chamber was filled with $600 \mu \mathrm{l}$ medium containing $10 \%$ FBS. Afterwards, the plate was put back into the $37^{\circ} \mathrm{C}$ incubator and the cells are allowed to grow freely. At $24 \mathrm{~h}$ post-seeding, the membrane was fixed with pre-cooled methanol and stained with crystal violet $(0.5 \%)$ for $5 \mathrm{~min}$. Cell migration ability was assessed by counting the cells that had migrated through the membrane. Five random fields of view were selected and images captured under a Nikon light microscope (Nikon Corporation, Tokyo, Japan) at a magnification of x100. For cell invasion 
A

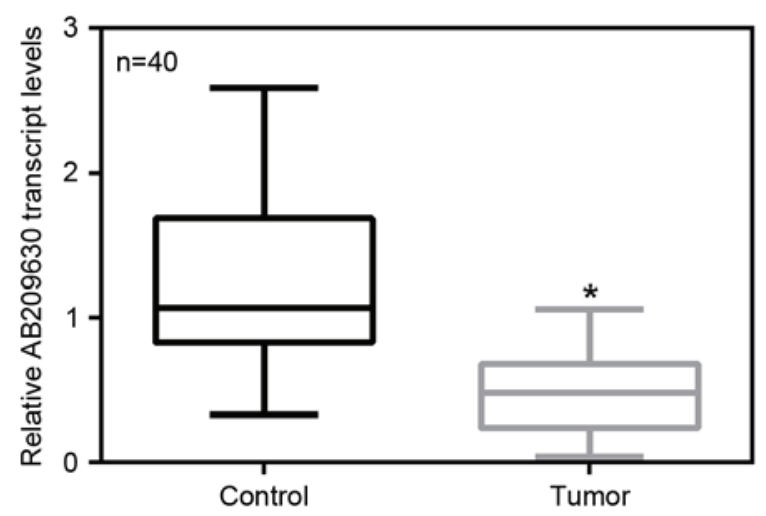

B

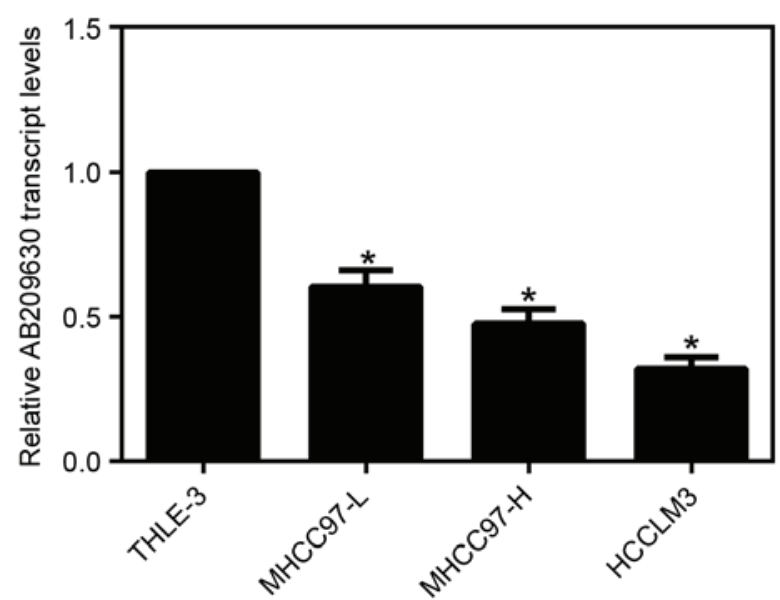

Figure 1. Expression of LncRNA AB209630 was downregulated in clinical HCC tissues and cultured HCC cells. (A) In 40 patients with HCC, the expression of AB209630 was significantly decreased in HCC tissues compared with the adjacent non-cancerous counterparts. ${ }^{*} \mathrm{P}<0.05$ vs. control. (B) Reverse transcription-quantitative polymerase chain reaction analysis revealed the relative transcript level of AB209630 was decreased in HCC cells compared with THLE-3 cells, a normal liver cell line. ${ }^{*} \mathrm{P}<0.05$ vs. THLE-3. LncRNA, long non-coding RNA; HCC, hepatocellular carcinoma.

assays, the membrane was pre-coated with Matrigel (Corning Incorporated, Corning, NY, USA) for $6 \mathrm{~h}$ at $37^{\circ} \mathrm{C}$ incubator.

Wound-healing assay. HCCLM3 cells were transfected with AB209630-expressing plasmid or control (empty) plasmid, cultured in DMEM in a six-well culture plate at a density of $5 \times 10^{5}$ cells/well and allowed to grow to a confluence of $90 \%$ overnight. The culture medium was replaced with serum-free DMEM. A line was scratched in the single cell layer using a 10- $\mu 1$ pipette tip and the cells were then washed with PBS three times. Following incubation for $24 \mathrm{~h}$, images of the migrating cells were observed and images captured using a light microscope.

Statistical analysis. In vitro experiments were repeated three times in triplicate, giving reproducible results. Results are presented as the mean \pm standard deviation following Student's t-test analysis. Data were analyzed using Prism 6 (GraphPad Software Inc., San Diego, CA, USA). P $<0.05$ was considered to indicate a statistically significant difference.

\section{Results}

Expression of LncRNA AB209630 is downregulated in clinical HCC tissues and cultured HCC cells. To examine the transcript levels of AB209630, 40 HCC patients from the People's Hospital of Weifang were included in the study. A tumor section and adjacent non-cancerous tissues were obtained from each patient. RT-qPCR analysis revealed a markedly lower expression of AB209630 in HCC tissues compared with the adjacent non-cancerous tissues (Fig. 1A).

MHCC97-L, MHCC97-H and HCCLM3 are three HCC cell lines, listed in order of increasing metastatic potential (19). The results shown in Fig. 1B indicate that the AB209630 transcript levels were decreased in the three HCC cell lines compared with those in THLE-3 normal liver cells, and were the lowest in HCCLM3 cells, which have the highest ability to transfer to other organs in patients. These data suggest that the transcript level of AB209630 was notably downregulated in HCC tissues and cultured HCC cells.

Construction of AB209630 plasmid successfully increases the expression of $A B 209630$ at the $m R N A$ and protein levels. To explore the role of decreased AB209630 expression in HCC cells, an expression plasmid containing the whole sequence of human AB209630 was prepared (17). Fig. 2A shows the pGLUE empty vector, in which the restriction enzyme EcoRV was used to insert the AB209630 sequence. The ability of the constructed plasmid to upregulate the expression of AB209630 was examined. In comparison with the untransfected control group, the transcript level of AB209630 was highly increased upon AB209630-expressing plasmid transfection but was not changed when the empty vector was transfected (Fig. 2B). Afterwards, western blot analysis was performed to detect the transfection efficiency of the AB209630-expressing plasmid. As shown in Fig. 2C, the protein level of the CBP tag was highly increased upon transfection with the AB209630-expressing plasmid compared with the control. These results verified that the AB209630-expressing plasmid was effective in promoting the transcription of AB209630.

Overexpression of AB209630 inhibits cell proliferation and colony formation in HCC cells. The effect of AB209630 on the proliferation of HCC cells was examined. After HCCLM3 cells were transfected with AB209630 plasmid, the proliferation rate remained unchanged in the following 3 days among the three experimental groups; however, on day 4, the proliferation rate of the HCCLM3 cells was inhibited by $42 \%$ in the AB209630-overexpressing group compared with the control group, and the inhibitory effect was even stronger on day 5 after transfection (Fig. 3A). A similar phenomenon was observed in the colony formation assays. In the control group, $>230$ colonies were formed 2 weeks post-seeding and there were 222 colonies in the vector group ( $\mathrm{P}>0.05)$; however, only 76 colonies were observed when AB209630 was overexpressed in HCCLM3 cells (Fig. 3B). These data reveal that the overexpression of AB209630 in HCCLM3 cells significantly suppressed cell proliferation.

Overexpression of AB209630 suppresses the migration and invasion of HCC cells. Transwell assays and wound-healing 


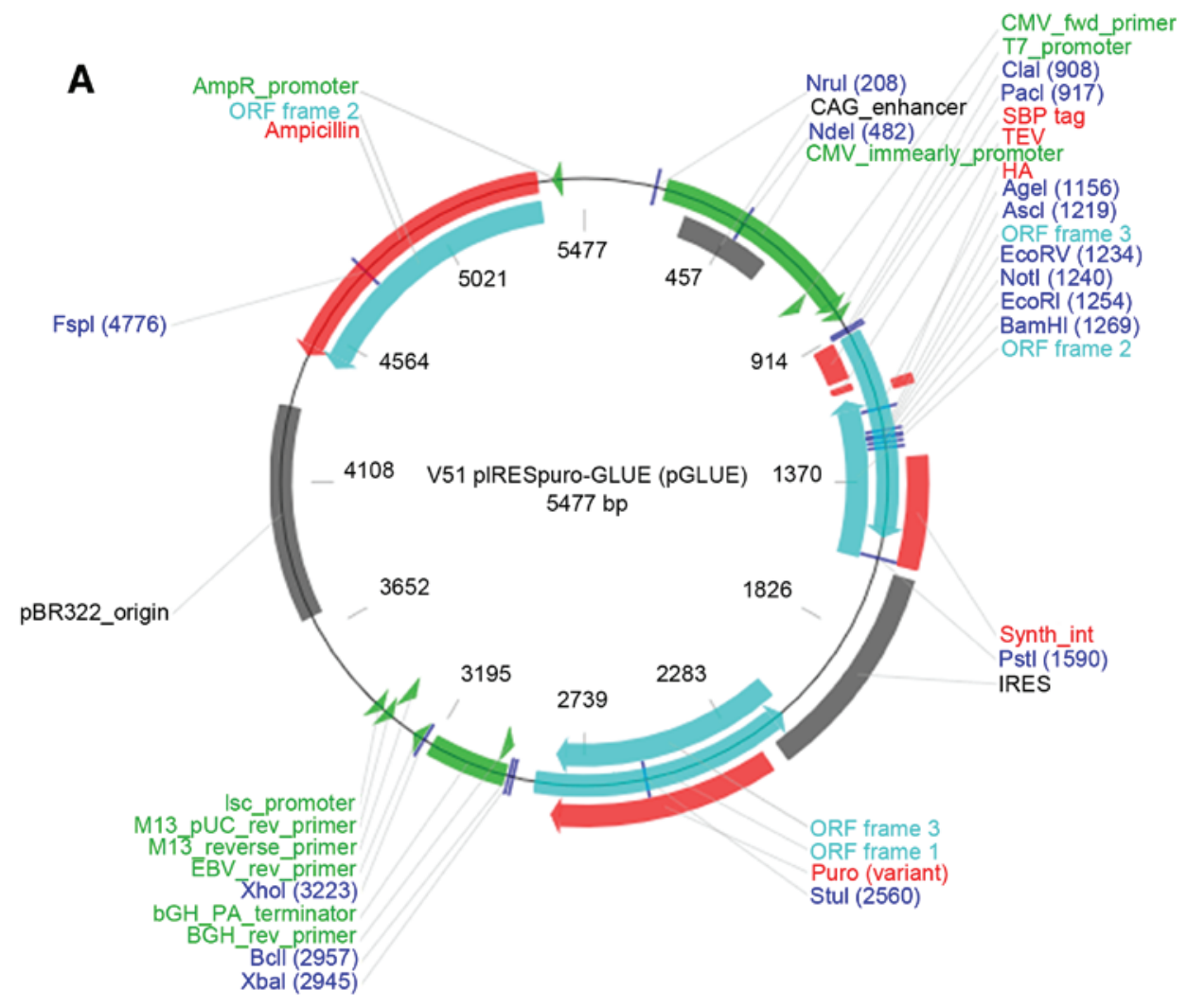

B

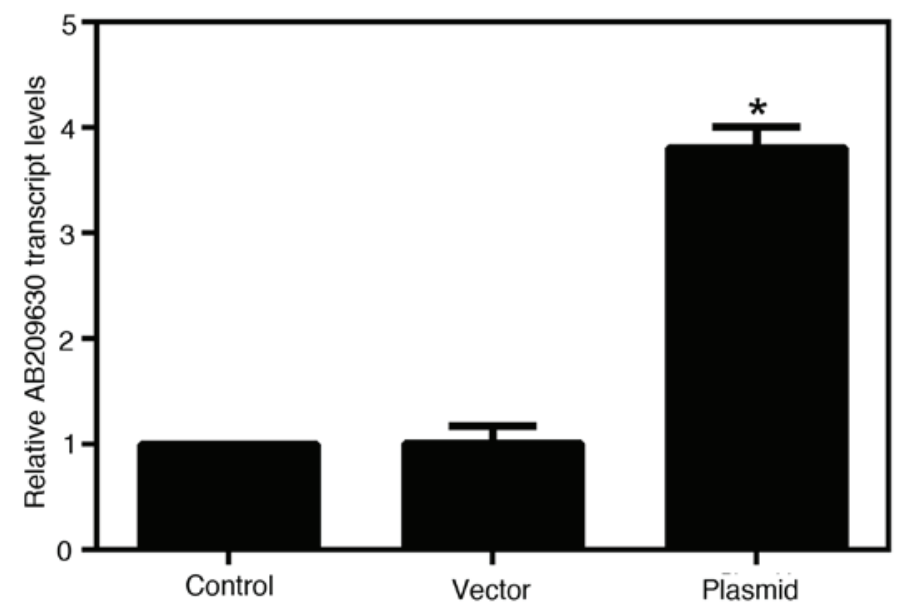

C

Control Vector Plasmid

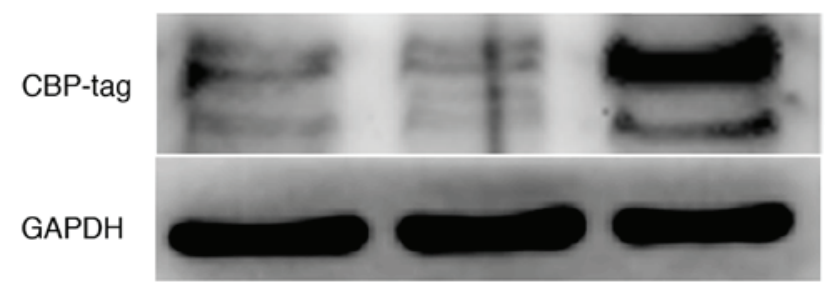

Figure 2. Construction of AB209630 plasmid successfully increased the expression of AB209630 at the mRNA and protein levels. (A) V51 pIRESpuro-GLUE (pGLUE) empty vector, used to clone the AB209630-expressing plasmid. Restriction enzyme EcoRV was used to insert AB209630. (B) Relative transcript levels of AB209630 in HCCLM3 cells transfected with the AB209630-expressing plasmid. "P<0.05 vs. control. (C) Western blot analysis showed the protein level of the CBP tag, which was connected to the inserted AB209630 transcript. GAPDH served as an internal control.

assays were performed to explore the role of AB209630 in cell migration. As shown in Fig. 4A, the upregulation of AB209630 reduced the migration of HCCLM3 cells through the membrane by $>70 \%$. Similarly, 200 untransfected HCCLM3 cells invaded to the lower surface of the membrane, but only 50 of the AB209630-upregulated cells did so (Fig. 4B). 
A

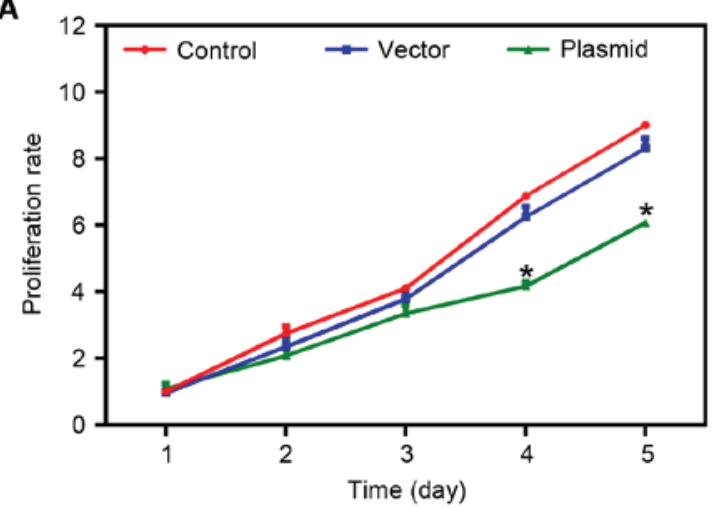

B

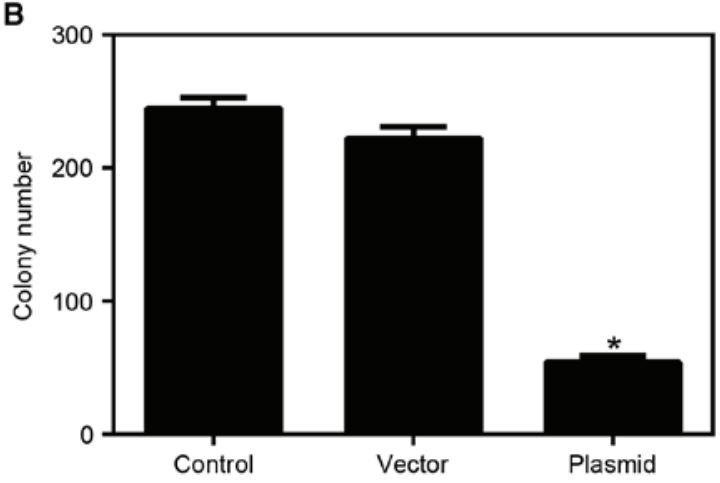

Figure 3. Overexpression of AB209630 inhibited cell proliferation and colony formation in HCC cells. (A) The cell proliferation rate was significantly inhibited upon AB209630 overexpression on days 4 and 5 after transfection. ${ }^{*} \mathrm{P}<0.05$ vs. control. (B) Colony formation assays were performed to assess the effects of AB209630 on HCCLM3 cells. The colonies were notably decreased in number when AB209630 was upregulated. ${ }^{*} \mathrm{P}<0.05$ vs. control. HCC, hepatocellular carcinoma.

Wound-healing assays demonstrated that cell migration was restrained by $55 \%$ in the AB209630-treated cells compared with the control cells (Fig. 4C). These results indicate that overexpression of LncRNA AB209630 suppressed the migration and invasion of HCC cells.

\section{Discussion}

Hepatocellular carcinoma (HCC) is the most prevalent malignancy of the liver and the second most common cause of cancer-related mortality, exceeded only by lung cancers (20). A wide variation in liver cancer incidence rates among nations has been observed worldwide, with a considerable geographic disparity in HCC (21). Notably, approximately half of all HCC cases and associated mortalities occur in China; thus, it is a significant health problem for people of different races and ethnicities, but particularly for Chinese individuals (22). The present study aimed to elucidate the role of LncRNA AB209630 in HCC, and to provide new information useful for the clinical diagnosis and treatment of HCC.

Three HCC cell lines, MHCC97-L, MHCC97-H and HCCLM3, were examined in present study. These cell lines are listed in order of increasing metastatic capacity, as established by the Liver Cancer Institute of Fudan University in 2001 (23). MHCC97 L was found to have a relatively low metastatic potential while MHCC97H and HCCLM3 were cell clones derived from MHCC97 cells that had incrementally
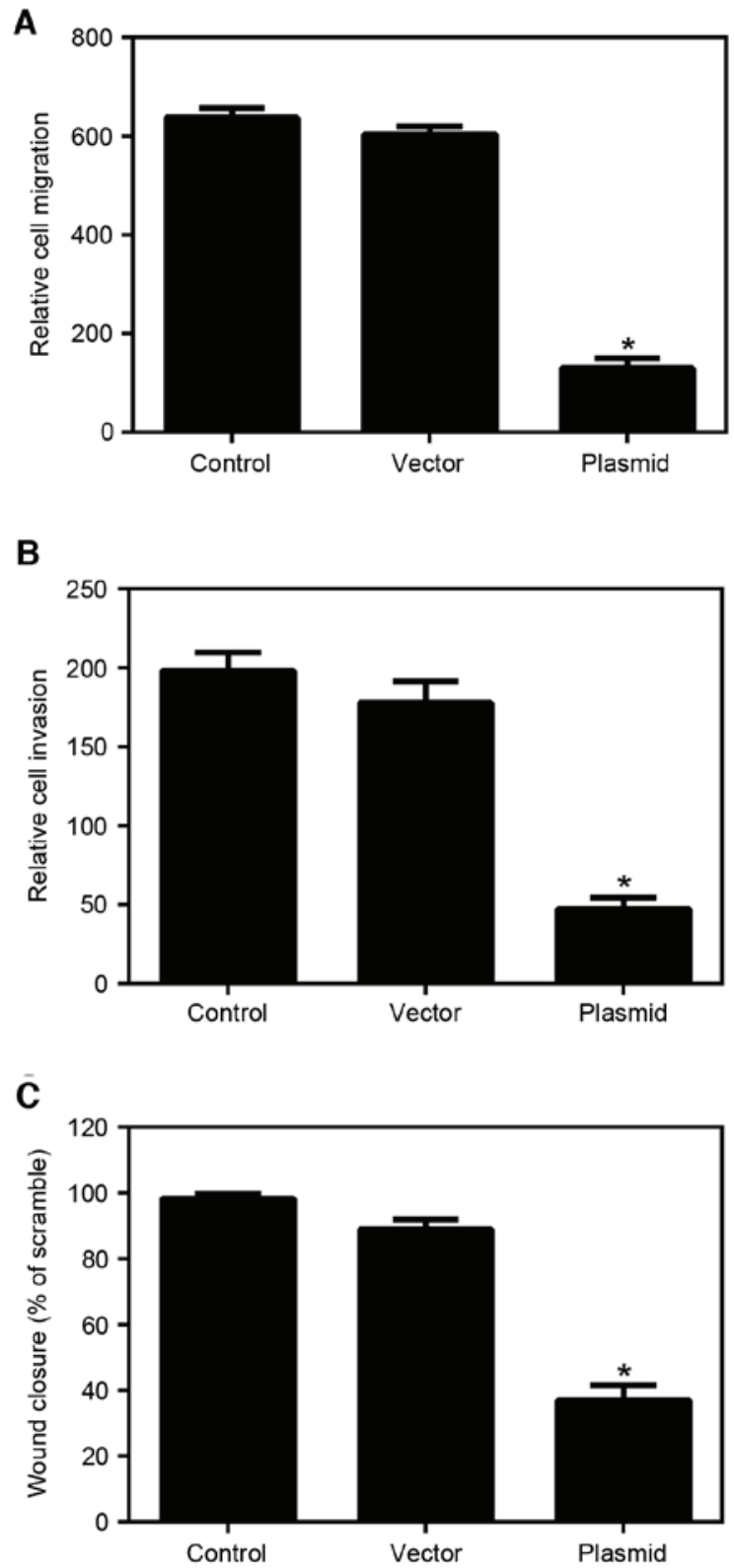

Figure 4. Overexpression of AB209630 suppressed the migration and invasion of HCC cells. (A) AB209630 overexpression inhibited the migration ability of HCCLM3 cells. (B) Cell invasion capacity was suppressed when cells were transfected with AB209630-expressing plasmid, compared with that of untransfected control cells. (C) Wound-healing assays showed cell migration was retarded by $>50 \%$ when cells were treated with AB209630 plasmid. ${ }^{*} \mathrm{P}<0.05$ vs. control. HCC, hepatocellular carcinoma.

higher metastatic capacity (23). Since considerable efforts are being made to identify metastasis-related genes, these three cell lines serve as useful materials for researchers. In the present study AB209630 expression was shown to be lower in HCC cells compared with the control liver cells (Fig. 1B). It was also observed that HCCLM3, the HCC cell line with the strongest potential for metastasis, had the lowest expression of AB209630, which further supported the conclusion that AB209630 is decreased in HCC cells and has a negative correlation with cell metastasis.

Another noteworthy feature of this study was the construction of an AB209630-expressing plasmid, which contained a CBP tag in the empty vector pGLUE. Since the transcript level 
of LncRNA AB209630 was decreased in HCC patients and cultured HCC cells, an AB209630-expressing plasmid (and not specific shRNA against AB209630), was used to transfect the cells. LncRNAs are non-coding RNAs, and so cannot be detected using western blot analysis. Thus, an empty vector containing a CBP tag was used, which enabled the expression of the CBP tag instead of the LncRNA to be detected. As shown in Fig. 2C, the protein expression of the CBP tag was significantly increased in the AB209630-expressing plasmid group, indicating that the plasmid was successfully transfected into the HCCLM3 cells. This method has also been described in previous literature (17,24-26). With the aid of AB209630-expressing plasmid, it was observed that overexpression of AB209630 significantly inhibited HCCLM3 cell proliferation and colony formation capacity. Likewise, it was further observed that the migration and invasion of HCCLM3 cells were also significantly inhibited by overexpression of AB209630. These functional assays led to the conclusion that AB209630 suppressed cell proliferation and metastasis in human HCC and the suggestion that AB209630 may be a promising tumor suppressor for HCC.

In conclusion, the present study revealed that LncRNA AB209630 was downregulated in HCC tissues and cultured HCC cells, the mechanism of which remains to be discovered. Overexpression of AB209630 inhibited the proliferation, migration and invasion of $\mathrm{HCC}$ cells, which is indicative of potential antimetastatic activity. AB209630 is a potential biomarker of $\mathrm{HCC}$ for use in clinical diagnosis and treatment, and may provide novel information useful for researchers involved in the study of HCC.

\section{References}

1. Yoon JH, Park JW and Lee JM: Noninvasive diagnosis of hepatocellular carcinoma: Elaboration on Korean Liver Cancer Study Group-National Cancer Center Korea Practice Guidelines compared with other guidelines and remaining issues. Korean J Radiol 17: 7-24, 2016

2. Sartorius K, Sartorius B, Aldous C, Govender PS and Madiba TE: Global and country underestimation of hepatocellular carcinoma (HCC) in 2012 and its implications. Cancer Epidemiol 39: 284-290, 2015

3. Han K, Kim JH, Ko GY, Gwon DI and Sung KB: Treatment of hepatocellular carcinoma with portal venous tumor thrombosis: A comprehensive review. World J Gastroenterol 22: 407-416, 2016.

4. Baghy K, Tatrai P, Regős E and Kovalszky I: Proteoglycans in liver cancer. World J Gastroenterol 22: 379-393, 2016.

5. Gelband H, Chen CJ, Chen W, Franceschi S, Hall SA, London WT, McGlynn KA, Wild CP: Liver Cancer. In: Cancer: Disease Control Priorities. Gelband H, Jha P, Sankaranarayanan R and Horton S (eds). Vol 3.3rd edition. The International Bank for Reconstruction and Development/The World Bank, Washington, DC, 2015.

6. Faloppi L, Scartozzi M, Maccaroni E, Di Pietro Paolo M, Berardi R, Del PM and Cascinu S: Evolving strategies for the treatment of hepatocellular carcinoma: From clinical-guided to molecularly-tailored therapeutic options. Cancer Treat Rev 37: 169-177, 2011.

7. Rahbari NN, Mehrabi A, Mollberg NM, Muller SA, Koch M, Büchler MW and Weitz J: Hepatocellular carcinoma: Current management and perspectives for the future. Ann Surg 253: 453-469, 2011.

8. Lou L, Ye W, Chen Y, Wu S, Jin L, He J, Tao X, Zhu J, Chen X, Deng A and Wang J: Ardipusilloside inhibits survival invasion and metastasis of human hepatocellular carcinoma cells. Phytomedicine 19: 603-608, 2012.

9. Lu W, Huang SY, Su L, Zhao BX and Miao JY: Long noncoding RNA LOC100129973 suppresses apoptosis by targeting miR-4707-5p and miR-4767 in vascular endothelial cells. Sci Rep 6: 21620, 2016.
10. Cesana M, Cacchiarelli D, Legnini I, Santini T, Sthandier O, Chinappi M, Tramontano A and Bozzoni I: A long noncoding RNA controls muscle differentiation by functioning as a competing endogenous RNA. Cell 147: 358-369, 2011.

11. Keniry A, Oxley D, Monnier P, Kyba M, Dandolo L, Smits G and Reik W: The H19 lincRNA is a developmental reservoir of miR-675 that suppresses growth and Igf1r. Nat Cell Biol 14: 659-665, 2012

12. Bertani S, Sauer S, Bolotin E and Sauer F: The noncoding RNA mistral activates Hoxa6 and Hoxa7 expression and stem cell differentiation by recruiting MLL1 to chromatin. Mol Cell 43: 1040-1046, 2011.

13. Gong C and Maquat LE: IncRNAs transactivate STAU1-mediated mRNA decay by duplexing with 3' UTRs via Alu elements. Nature 470: 284-288, 2011.

14. Clemson CM, Hutchinson JN, Sara SA, Ensminger AW, Fox AH, Chess A and Lawrence JB: An architectural role for a nuclear noncoding RNA: NEAT1 RNA is essential for the structure of paraspeckles. Mol Cell 33: 717-726, 2009.

15. Zhou T and Gao Y: Increased expression of LncRNA BANCR and its prognostic significance in human hepatocellular carcinoma. World J Surg Oncol 14: 8, 2016.

16. EspostiDD,Hernandez-Vargas H,VoegeleC,Fernandez-JimenezN, Forey N, Bancel B, Le Calvez-Kelm F, McKay J, Merle P and Herceg Z: Identification of novel long non-coding RNAs deregulated in hepatocellular carcinoma using RNA-sequencing. Oncotarget 7: 31862-31877, 2016.

17. Zhou J, Li M, Yu W, Li W, Wang J, Xiang X, Li G, Pan X and Lei D: AB209630 a long non-coding RNA decreased expression in hypopharyngeal squamous cell carcinoma, influences proliferation invasion metastasis and survival. Oncotarget 7: 14628-14638, 2016.

18. Livak KJ and Schmittgen TD: Analysis of relative gene expression data using real-time quantitative PCR and the 2(-Delta Delta C(T)) method. Methods 25: 402-408, 2001.

19. Li Y, Tang Y, Ye L, Liu B, Liu K, Chen J and Xue Q: Establishment of a hepatocellular carcinoma cell line with unique metastatic characteristics through in vivo selection and screening for metastasis-related genes through cDNA microarray. J Cancer Res Clin Oncol 129: 43-51,2003.

20. Waly Raphael S, Yangde Z and Yuxiang C: Hepatocellular carcinoma: Focus on different aspects of management. ISRN Oncol 2012: 421673, 2012.

21. Blonski W, Kotlyar DS and Forde KA: Non-viral causes of hepatocellular carcinoma. World J Gastroenterol 16: 3603-3615, 2010.

22. Kim DY and Han KH: Epidemiology and surveillance of hepatocellular carcinoma. Liver Cancer 1: 2-14, 2012.

23. Li Y, Tang ZY, Ye SL, Liu YK, Chen J, Xue Q, Chen J, Gao DM and Bao WH: Establishment of cell clones with different metastatic potential from the metastatic hepatocellular carcinoma cell line MHCC97. World J Gastroenterol 7: 630-636, 2001.

24. Sidi AA, Ohana P, Benjamin S, Shalev M, Ransom JH, Lamm D, Hochberg A and Leibovitch I: Phase I/II marker lesion study of intravesical BC-819 DNA plasmid in H19 over expressing superficial bladder cancer refractory to bacillus Calmette-Guerin. J Urol 180: 2379-2383, 2008.

25. Dong S, Qu X, Li W, Zhong X, Li P, Yang S, Chen X, Shao M and Zhang L: The long non-coding RNA GAS5 enhances gefitinib-induced cell death in innate EGFR tyrosine kinase inhibitor-resistant lung adenocarcinoma cells with wide-type EGFR via downregulation of the IGF-1R expression. J Hematol Oncol 8: 43, 2015.

26. DeOcesano-Pereira C, Amaral MS, Parreira KS, Ayupe AC, Jacysyn JF, Amarante-Mendes GP, Reis EM and Verjovski-Almeida S: Long non-coding RNA INXS is a critical mediator of BCL-XS induced apoptosis. Nucleic Acids Res 42: 8343-8355, 2014. 